\title{
Ethyl-3,4-dephostatin Inhibits PTPN2 and Induces ERK Activation
}

\author{
Huiyun Seo and Sayeon Cho* \\ College of Pharmacy, Chung-Ang University, Seoul 156-756, Korea. *E-mail: sycho@cau.ac.kr \\ Received May 13, 2011, Accepted May 27, 2011
}

Key Words : PTPN2, Ethyl-3,4-dephostatin, PTP inhibitor

The mitogen-activated protein kinases (MAPKs) are key signaling enzymes that play critical roles in cellular signal transduction pathways such as cell survival, proliferation, differentiation, and apoptosis. The three major groups of MAPK that are expressed in mammals are p38, extracellular signal regulated kinase (ERK), c-Jun N-terminal kinase (JNK) ${ }^{1,2}$ Signaling by MAPKs is processed following their phosphorylation at conserved threonine and tyrosine residues within the activation loop of MAPKs mediated by their upstream kinases in activation. ${ }^{3}$

Protein tyrosine phosphatases (PTPs) are responsible for regulation of MAPK activity through dephosphorylation of phospho-threonine and phospho-tyrosine residues on MAPKs. ${ }^{4}$ The human genome encodes 107 PTPs. PTPs can be classified into four groups based on amino-acid sequences of catalytic domains. They are class I Cys-based PTPs, class II Cys-based PTPs, class III Cys-based PTPs, and Asp-based PTPs. ${ }^{5}$ Especially, protein tyrosine phosphatase non-receptor type 2 (PTPN2) that is also known as T cell protein tyrosine phosphatase (TC-PTP) belongs to class I Cys-based PTPs as the intracellular, non-receptor PTPs (NRPTPs) with a high degree of sequence and structural homology within the catalytic domain. ${ }^{6,7}$ PTPN2 has been reported to modulate cytokine receptor signaling, including IFN- $\gamma$ signaling., PTPN2 dephosphorylates and inactivates Src tyrosine kinases to suppress downstream signaling through ERK and production of interleukin $6 .^{10}$

In a way to study biological functions of PTPN2, we performed the regulatory mechanism of PTPN2 activity using a specific chemical compound, ethyl-3,4-dephostatin. Ethyl-3,4-dephostatin (Fig. 1) was originally identified as a potent PTP-1B and src homology-2-containing protein tyrosine phosphatase-1 (SHP-1) inhibitor and showed inhibitory effect on several PTPs in vitro. ${ }^{11,12}$ Recently, dual-specificity phosphatase 22 (DUSP22) was identified as an additional target of ethyl-3,4-dephostatin. ${ }^{13}$

To investigate whether ethyl-3,4-dephostatin was able to inhibit PTPN2 activity, we treated various concentrations of ethyl-3,4-dephostatin on purified active PTPN2. PTPN2

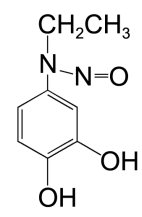

Figure 1. Chemical structure of ethyl-3,4-dephostatin. activity was decreased by ethyl-3,4-dephostatin in a dosedependent manner. The half maximal inhibitory concentration $\left(\mathrm{IC}_{50}\right)$ of PTPN2 was found to be about $6.5 \pm 0.54 \mu \mathrm{M}$ by using the curve fitting program PRISM 3.0 (Fig. 2(a)). In addition, the Lineweaver-Burk plot shows that the $K_{\mathrm{i}}$ was 8.6 $\mu \mathrm{M}$ (Fig. 2(b)). This result indicates that ethyl-3,4dephostatin acts as a competitive inhibitor of PTPN2, suggesting that ethyl-3,4-dephostatin suppresses the activity of PTPN2 through binding to the catalytic site.

We further examined whether ethyl-3,4-dephostatin influences PTPN2-mediated ERK inhibition in vivo. Since PTPN2 inactivates Src tyrosine kinases that are located upstream of ERK signaling, we overexpressed PTPN2 in HEK 293 cells and detected phosphorylation levels of endogenous ERK after treatment with ethyl-3,4-dephostatin. HEK 293 cells were transfected with FLAG-PTPN2 expression plasmid and

(a)

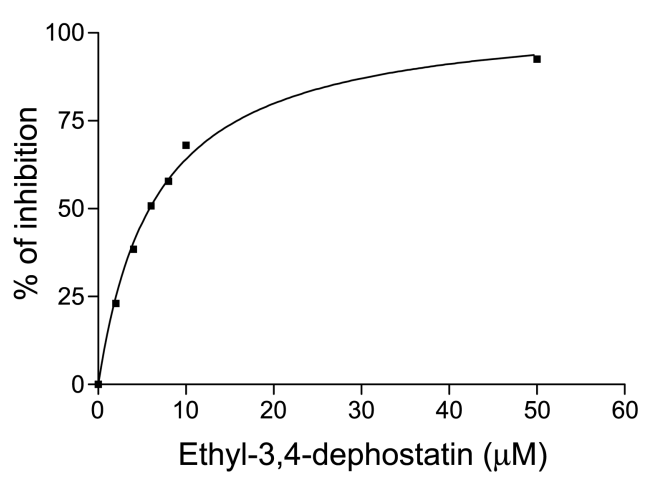

(b)

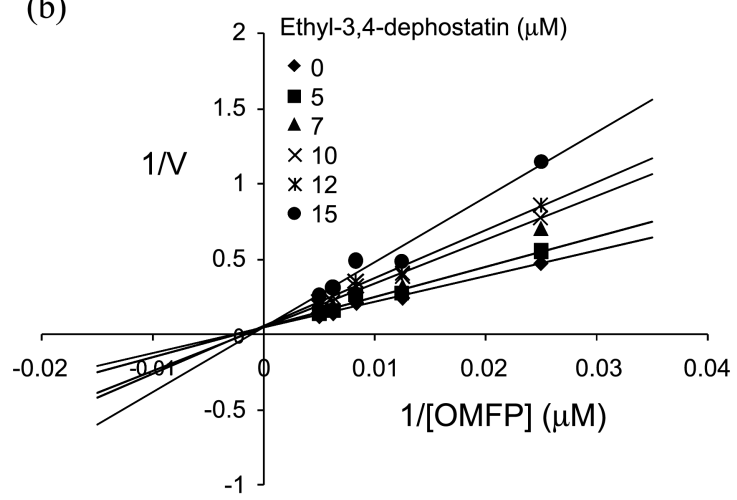

Figure 2. Kinetic analysis of PTPN2 inhibition by ethyl-3,4-dephostatin. (a) PTPN2 was incubated with various concentrations of the inhibitor. Assays were carried out as described in Experimental Section. (b) Lineweaver-Burk plots of PTPN2 were generated from the reciprocal data. 


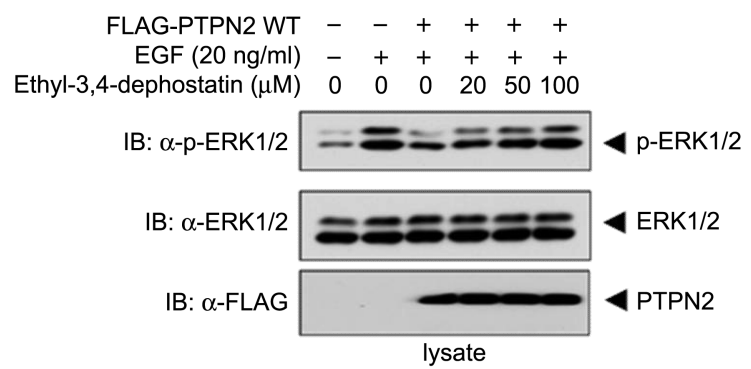

Figure 3. In vivo effect of ethyl-3,4-dephostatin on PTPN2regulated phospho-ERK. HEK 293 cells were transfected with $1 \mu \mathrm{g}$ of PTPN2 expression plasmids. After $48 \mathrm{~h}$ of transfection, HEK 293 cells were pretreated with various concentrations of ethyl-3,4dephostatin $(0,20,50$, or $100 \mu \mathrm{M})$ for $3 \mathrm{~h}$ and then stimulated with EGF (20 ng/mL, $10 \mathrm{~min})$. Cell lysates were analyzed by immunoblotting with appropriate antibodies.

pretreated with various concentrations of ethyl-3,4-dephostatin for $3 \mathrm{~h}$ and then stimulated with EGF for $10 \mathrm{~min}$ to phosphorylate endogenous ERK. The cells were lysed with PTP lysis buffer. Lysates were subjected to SDS-PAGE and then immunoblotted with anti-phospho-ERK, anti-ERK, and anti-FLAG antibodies, respectively (Fig. 3). The result shows that the phospho-ERK levels are efficiently recovered from PTPN2-induced dephosphorylation of phospho-ERK by ethyl-3,4-dephostatin.

In this study, we verified ethyl-3,4-dephostatin as a potent PTPN2 inhibitor and identified that its inhibitory ability protects ERK activity from PTPN2 in cells. In addition, it has been reported that a negative correlation between sensitivity to IFN- $\gamma$ and PTPN2 activity has been shown in the case of chronic myelogenous leukemia. ${ }^{14,15}$ Therefore, this study for ethyl-3,4-dephostatin against PTPN2 will have a beneficial effect in several PTPN2-related diseases such as chronic myelogenous leukemia.

\section{Experimental Section}

Reagents and Antibodies. Anti-ERK, anti-phospho-ERK (specific for phospho-Thr202 and phospho-Tyr204) antibodies, and active ERK protein were purchased from Cell Signaling Technology. EGF was purchased from SigmaAldrich (E4269). LipofectAMINE used for transfection into HEK 293 cell was purchased from Invitrogen.

Cell Culture and Transfection. Human embryonic kidney (HEK) 293 cells were maintained at $37^{\circ} \mathrm{C}$ in Dulbecco's modified Eagle's medium (DMEM, Invitrogen, Carlsbad California) supplemented with $10 \%$ fetal bovine serum (FBS, Invitrogen) and penicillin/streptomycin in the presence of $5 \% \mathrm{CO}_{2}$. For transient transfection, $1.4 \times 10^{6}$ cells were plated in each $60 \mathrm{~mm}$ cell culture plate, grown overnight, and transfected with DNA using LipofectAMINE.

Plasmid Constructions. FLAG-tagged PTPN2 expression plasmid was constructed in pcDNA3.1 (Invitrogen). Histagged PTPN2 plasmid was constructed in pET28a plasmid (Novagen, Darmstadt, Germany) for protein expression in Escherichia coli.
Purification of the six-His-tagged PTPN2 Phosphatase. Purification of the six-His-tagged PTPN2 was carried out as previously described. ${ }^{13}$

In vitro Phosphatase assays and Kinetic Analysis. In vitro phosphatase assays and kinetic analysis were carried out as previously described. ${ }^{13}$

Immunoblotting Analysis. After HEK 293 cells were cotransfected with PTPN2 expression plasmids for $48 \mathrm{~h}$, cells were washed twice with phosphate buffered saline (PBS) buffer and lysed in PTP lysis buffer (0.5\% NP-40, $0.5 \%$ Triton X-100, $150 \mathrm{mM} \mathrm{NaCl}, 20 \mathrm{mM}$ Tris- $\mathrm{HCl}(\mathrm{pH}$ 8.0), $1 \mathrm{mM}$ EDTA, 1\% glycerol, $1 \mathrm{mM}$ phenylmethylsulfonyl fluoride, and $1 \mu \mathrm{g} / \mathrm{mL}$ aprotinin) for $30 \mathrm{~min}$ at $4{ }^{\circ} \mathrm{C}$. Cleared cell lysates from centrifugation were mixed with protein sample buffer, boiled at $100{ }^{\circ} \mathrm{C}$ for $5 \mathrm{~min}$, subjected to SDSPAGE, and subsequently transferred onto nitrocellulose (NC) membrane. The membranes were then blocked with $5 \%$ skim milk/TBS for $1 \mathrm{~h}$ and incubated with the appropriate primary antibodies and HRP-conjugated secondary antibodies at room temperature. Following extensive washing, protein bands were visualized using enhanced chemiluminescence Western blotting detection reagents (Pierce, Rockford, IL, USA).

Inhibition Study. The inhibition constant $\left(K_{i}\right)$ to PTPN2 for the inhibitor was determined by measuring the initial rates at several OMFP concentrations for each fixed concentration of the inhibitor. The data were fitted to the following equation to obtain the inhibition constant of reversible competitive inhibitors. The slopes obtained were replotted against the inhibitor concentrations. The $K_{i}$ value was obtained from the slopes of these plots. ${ }^{16}$

$$
1 / \mathrm{V}=K_{m}\left(1+[\mathrm{I}] / K_{i}\right) \mathrm{V}_{\max }[\mathrm{S}]+1 / \mathrm{V}_{\max }
$$

Acknowledgments. This work was supported by the Korea Science and Engineering Foundation (KOSEF) grant funded by the Korea government (MEST) (No. 20110003139).

\section{References}

1. Chang, L.; Karin, M. Nature 2001, 410, 37.

2. Schaeffer, H. J.; Weber, M. J. Mol. Cell Biol. 1999, 19, 2435.

3. Theodosiou, A.; Ashworth, A. Genome Biol 2002, 3, REVIEWS3009.

4. Stoker, A. W. J. Endocrinol. 2005, 185, 19.

5. Alonso, A.; Sasin, J.; Bottini, N.; Friedberg, I.; Friedberg, I.; Osterman, A.; Godzik, A.; Hunter, T.; Dixon, J.; Mustelin, T. Cell 2004, 117, 699.

6. Asante-Appiah, E.; Ball, K.; Bateman, K.; Skorey, K.; Friesen, R.; Desponts, C.; Payette, P.; Bayly, C.; Zamboni, R.; Scapin, G.; Ramachandran, C.; Kennedy, B. P. J. Biol. Chem. 2001, 276, 26036.

7. Jia, Z.; Barford, D.; Flint, A. J.; Tonks, N. K. Science 1995, 268, 1754.

8. Myers, M. P.; Andersen, N. K.; Cheng, A.; Tremblay, M. L.; Horvath, C. M.; Parisien, J. P.; Salmeen, A.; Barford, D.; Tonks, N. K. J. Biol. Chem. 2001, 276, 47771.

9. Simoncic, P. D.; Lee-Loy, A.; Barber, D. L.; Tremblay, M. L.; McGlade, C. J. Curr. Biol. 2002, 12, 446.

10. van Vliet, C.; Bukczynska, P. E.; Puryer, M. A.; Sadek, C. M.; Shields, B. J.; Tremblay, M. L.; Tiganis, T. Nature Immunology 
$\mathbf{2 0 0 5}, 6,253$

11. Suzuki, T.; Hiroki, A.; Watanabe, T.; Yamashita, T.; Takei, I.; Umezawa, K. J. Biol. Chem. 2001, 276, 27511.

12. Watanabe, T.; Suzuki, T.; Umezawa, Y.; Takeushi, T.; Otsuka, M.; Umezawa, K. Tetrahedron 2000, 56, 741.

13. Seo, H.; Kim, H.; Cho, S. Bulletin of the Korean Chemical Society 2011, 32, 1379.
14. Shimizu, T.; Miyakawa, Y.; Iwata, S.; Kuribara, A.; Tiganis, T.; Morimoto, C.; Ikeda, Y.; Kizaki, M. Exp. Hematol. 2004, 32, 1057.

15. Shimizu, T.; Miyakawa, Y.; Oda, A.; Kizaki, M.; Ikeda, Y. Exp. Hematol. 2003, 31, 601.

16. Shi, Z.; Tabassum, S.; Jiang, W.; Zhang, J.; Mathur, S.; Wu, J.; Shi, Y. Chembiochem. 2007, 8, 2092. 